\title{
Fusion of Ear and Soft-biometrics for Recognition of Newborn
}

\author{
Shrikant Tiwari, Aruni Singh, Sanjay Kumar Singh \\ Department of Computer EngineeringInstitute of Technology, Banaras Hindu University \\ Varanasi, India-221005 \\ shrikant.rs.cse@itbhu.ac.in, arunisingherocketmail.com, \\ sks.cseditbhu.ac.in
}

\begin{abstract}
Recognition of newborn after birth is a critical issue for hospitals, maternity word and other places where multiple birthstake place. Switching and abduction of newborn is a global challenge and research done to meet this challenge is minimal. Most of the biometric systems developed are for adults and very few of them address the issue of newborn identification.The ear of newborn is a perfect source of data for passive identification of newborn as they are the highly non cooperative users of biometrics. The four important characteristics of ear biometrics: universality, uniqueness, permanence and collectability make it a very potential biometric trait for the identification of newborn. Further the use of soft-biometric data like gender, blood group, height and weight along with ear enhances the accuracy for identification of newborn. The objective of this paper is to demonstrate the concept of fusing earand soft-biometrics for recognition of newborn. The main contributions of the research are (a) Design and implementation for fusion of ear and soft biometric for recognition of 210 newborn. (b)Preparation of ear and soft-biometric database of newborn. Fusion of ear and soft-biometrics results in an improvement of approximately 5.59\% over the primary biometric system i.e. ear.
\end{abstract}

\section{KEYWORDS}

Ear, Soft-biometric, Newborn, Recognition, Fusion

\section{INTRODUCTION}

Reliability and efficiency for newborn recognition are key to the stringent security requirements to control mixing, swapping, kidnapping and illegal adoption of newborn. The level of security is very crucial issue in maternity ward and the problem of missing and swapping of newborn is of prime concern to the persons involved and affected. There is a common perception in the society that they are helpless and they can do nothing to prevent this unfortunate event. In comparison to developed nations the developing countries are facing more challenges because of overcrowding and scarcity of medical facilities in the hospital. According to study every year around $1,00,000$ to 5,00,000 newborns in United States are exchanged (swapped) by mistake, or one out of every eight babies born in American hospitals sent home with the wrong parents [1]. According to report, out of 34 newborns that are admitted to a neonatal intensive care unit there are $50 \%$ probabilities of incorrect newborns identification only in a single day $[2,3]$. These are the number of cases that have been reported, but there may be many more cases that are undeclared or the parents and the children never come to know about this unfortunate incident.

DOI : $10.5121 /$ sipij.2012.3309 
Hospitals have devised different procedures to ensure that babies are correctly recognized and one of the popular methods is the use of ID bracelets. Soon after the birth ID bracelets are put on babies hands/legs, but this has not been able to provide enough level of security for newborn. The medical technique like Deoxyribonucleic Acid (DNA) typing and Human Leukocyte Antigen (HLA) typing are very efficient and accurate methods for verifying the identity of babies but due to the amount of time it takes to process a DNA or HLA sample and the cost associated with it, these methods are not feasible for every newborn. Other popular method recommended by Federal Bureau of Investigation is foot and finger printing of the child and mother [4]. According to survey report $90 \%$ of the hospitals in United States perform foot printing of the babies within 2 hours of their birth and hospitals maintain newborn identification form on which footprint of the child and fingerprint of the mother are collected. The prints are generally collected using ink based methods and then printed on the identification form.Medical and computer scientist have explored the efficiency and authenticity of using footprints for newborn identification and analysis done by Shepard et al. using footprints of 51 newborns was examined by fingerprint experts ant they were able to identified only 10 newborn $[5,6]$.

According to study [7] on1917 foot prints collected by trained staff of hospital in Brazil, where most of the images collected provided insufficient information for identification of newborn [7]. Researchers also explored the applicability of other biometric modalities such as fingerprint, palm print and ear for verifying the identity of newborn babies [8]. Weingaertner et al. developed a new high resolution sensor for capturing the foot and palm prints of babies [9]. Two images of 106 newborns were collected: one within 24 hours of birth and another at around 48 hours. Fingerprint experts examined the data and the identification accuracy of $67.7 \%$ and $83 \%$ were obtained using foot prints and palm prints respectively. However, multiple studies have quoted that capturing finger/palm/footprint of newborns is very challenging as it is difficult to hold their hands and legs still. Fields et al. have studied the feasibility of ear recognition on a database of 206 newborns [10]. They manually analyzed the samples and concluded that visually ears can be used to distinguish between two children. In all the methods for identifying newborns, no research has evaluated the performance of automatic identification or verification.

Another biometric modalities that have been extensively studied for adults are face and iris recognition [11,12]. Although iris recognition for adults yields very high accuracy, for newborns, it is very difficult to capture iris patterns [12]. The work done on face recognition of newborn reports the accuracy of $86.9 \%$ on the database of 34 babies also suffers from facial expression of newborn as the face database consist of crying or sleeping face because it is very difficult to get the normal face[13]. The work done by Rubisley P Lemes et al. demonstrate the use of palmprint using high resolution scanner on the database of 250 newborn has the limitation of good quality image and high cost recognition [14].

Another work done by Tiwari et. al. tested the identification accuracy of newborn using ear. The result on database of 210 newborn using Geometrical Feature Extraction (GF) is $83.67 \%$, but the limitation of using ear biometrics for newborn is pose and illumination covariates [15].Recently work done Tiwari et.al. tested the identification performance of newborn combining with face and soft-biometrics on the newborn database [16]. Shorter version of this manuscript is published in the proceedings of SPPR-2012[17]. The rest of the paper is organised as follows: The section 2 outlines the motivation for ear as biometric for newborn. This section is followed by details of database description in section 3. The details of feature extraction and matching and fusion of ear 
and soft-biometric information are explained in section 4. Finally section 5 and 6 present future direction and conclusion of the paper.

\section{MOTIVATION}

The motivation behind selection of ear for newborn recognition is due to robustness of the ear shape $[18,19,20,21,22,23]$. They have distinguishing and stable feature that changes little with age. The limitation of face biometrics compared to ear is that it does not suffer from changes in facial expression. In case of newborn ear biometric is better than other biometrics specially face because most of the time newborn are either sleeping or crying and thus it is not affected by any expression. In comparison to biometric trait like iris, retina and fingerprint ear is bigger are size and the capturing of image can be easily done at a distance.It is our opinion that ear recognition can be a hygienic, friendly and cost effective solution for identifying newborns if the performance of automatic matching algorithms is satisfactory. In this research, we have investigated the applicability and performance of ear recognition to prevent newborn switching, illegal adoption and abduction.

Soft Biometrics characteristics like gender, blood-group, age, height, weight and head print are not unique and reliable but they provide some useful information about the individual and these are referred as soft biometric trait and these trait compliment the primary biometric trait $[24,25$, $26,27]$. Soft biometric traits help in filtering large databases by reducing the number of search for each query. In case of newborns we have collected gender, height, weight and blood group as soft-biometric data. Biometric system based on a single source of biometric information are called unimodal biometric system fail due to noisy sensor data, non universality, large intra user variations, lack of individuality of the chosen biometric trait, susceptibility to spoof attacks and poor error rates. So some of these problems can be alleviated by using ear and soft-biometrics that fuse evidence from face and soft-biometrics of the same newborn.

\section{DATABASE DESCRIPTION}

The primary reason of limited research for newborn identification is the non availability of reference database in public domain. The main problem in preparing the database of newborn is the consent of parents and the cooperation of medical staff to prepare the database. For the successful preparation of the newborn database the active participation of parents and the medical staff provides an additional advantage. It is really difficult to convince the parents for data acquisition as some parents were unwilling and concerned about the privacy issue. New born are highly uncooperative users of biometrics and most of the time they are sleeping or crying. Therefore, to capture their image is really a difficult task because as soon as they are targeted for data acquisition they get disturbed and start crying. During biometric data acquisition a crucial problem faced by the biometric researchers is to decide an opportune time of the data acquisition. If a newborn is uncomfortable due to hunger or medical illness then he/she will cry and ceaselessly move his/her head, feet or whole body. Even if they are sleeping, then the task of their data acquisition becomes more challenging.

The newborns database consists of static digital images of ear (Digital camera of 10megapixels and video camera of 14 megapixels to capture the images of ear) and soft biometrics data like gender, height, weight and blood group. The data base acquisition of newborns took one year to 
complete and thus it has minor variations in pose, expression and certain illumination variations due to the newborns movement, some instances of motion blurriness also present in the newborn database.The ear database of newborn consists of pose, illumination and occlusion covariates. Occlusion is due to some tradition that soon after birth parents put black earrings or black threads in the ear. Ear images are grouped according to variations mentioned above to solve the problem of ear recognition in newborn as shown in Figure 1 (a), Figure 1(b) and Figure 1(c).

Ear recognition is a long studied problem and several challenges have been identified by the researchers including pose, illumination, and occlusion. Since it is difficult to make the newborns sit still and give good ear images, they can be considered as uncooperative users of biometric recognition. They may also exhibit different poses, especially if they become uncomfortable while capturing the ear image. In some cases occlusion is also an important issue because soon after the birth some parents put black thread or ear ring due to their tradition. But for our experiment, we have tried to use good ear images with less covariate.

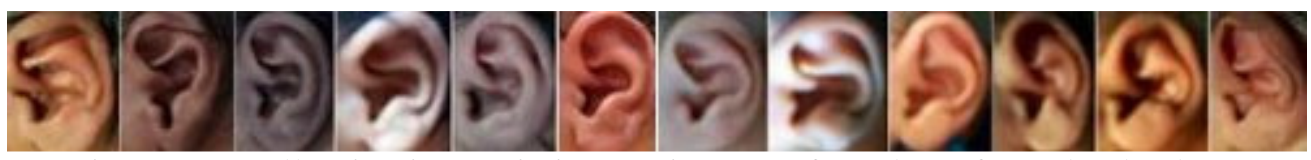

Figure 1 (a). Illumination variation ear images of newborn from the database

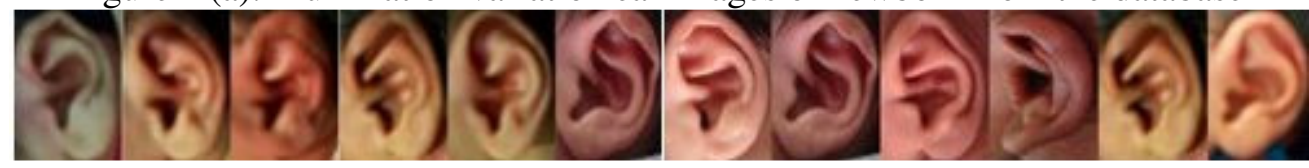

Figure 1 (b). Pose variation ear images of newborn from the database

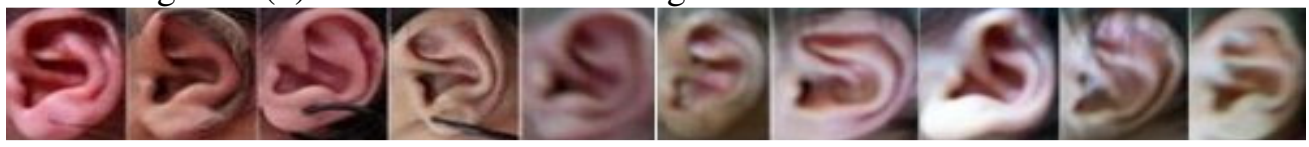

Figure 1 (c). Occlusion variation ear images of newborn from the database

The datasbase of newborn includes 2100 images of ear from 210 subjects with 10 images per person out of which first 4 images of each newborn is randomly selected for training/gallery database (total of 840 images) and the remaining 6 images of each newborn is selected for testing/probe database (total 1260 images). In the pre-processing step the ear part is manually cropped color images to a size of $1402 \times 1900$ pixels as shown in Figure2(b). The cropped color image is converted to a gray scale image as shown in Figure 2(c). The normalization of ear image is done in two stages. The geometric normalization scales all the images to the standard size of $160 \times 160$. In photometric normalization different levels of masking are experimented for finding the best one to get as good accuracy as possible for the algorithm.
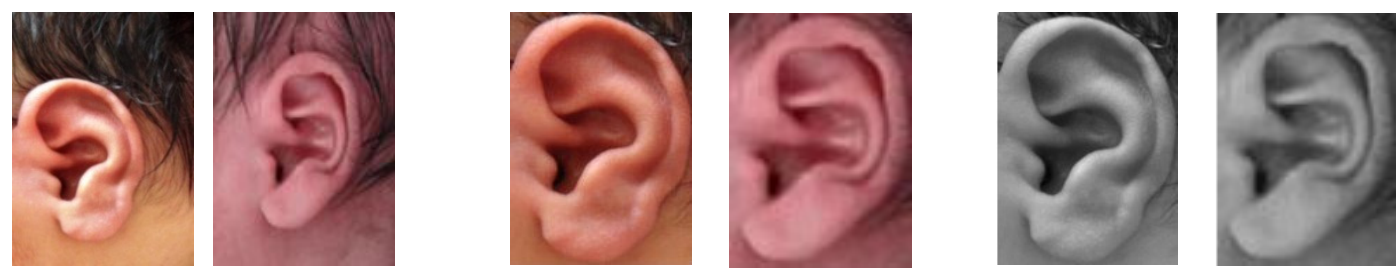

Figure2 (a). Before Cropping Figure2 (b). After Cropping Figure 2 (c). Normalized Ear Image

The weight of an newborn is measured by digital weighting machine at the place where the newborn lie while providing the primary biometric. The height can be estimated from 
Infantometer obtained when the newborn coming for checkup. The Figure 3 displays a mechanism for capturing of the height and weight information of an newborn. These images are captured without imposing any constraint on the newborn or their surroundings. The database statistics of Soft-biometrics is shown in the Table 1.

Table 1: Database Statistics of Soft Biometrics

\begin{tabular}{|c|c|c|c|c|c|c|c|c|}
\hline \multirow{3}{*}{$\begin{array}{l}\text { Gender } \\
\text { Distribution } \\
\text { Blood Group }\end{array}$} & \multicolumn{4}{|c|}{ Male } & \multicolumn{4}{|c|}{ Female } \\
\hline & \multicolumn{4}{|c|}{70} & \multicolumn{4}{|c|}{140} \\
\hline & $\mathrm{A}+$ & A- & $\mathrm{B}+$ & B- & $\mathrm{AB}+$ & AB- & $\mathrm{O}+$ & $\mathrm{O}-$ \\
\hline & 32 & 21 & 30 & 20 & 25 & 18 & 58 & 6 \\
\hline \multirow[t]{2}{*}{ Height } & \multicolumn{3}{|c|}{$40 \mathrm{~cm}$ to $45 \mathrm{~cm}$} & \multicolumn{2}{|c|}{$46 \mathrm{~cm}$ to $50 \mathrm{~cm}$} & \multicolumn{3}{|c|}{ more than $51 \mathrm{~cm}$} \\
\hline & \multicolumn{3}{|c|}{50} & \multicolumn{2}{|c|}{130} & \multicolumn{3}{|c|}{30} \\
\hline \multirow[t]{2}{*}{ Weight } & \multicolumn{3}{|c|}{$1500 \mathrm{gm}$ to $2500 \mathrm{gm}$} & \multicolumn{2}{|c|}{$2501 \mathrm{gm}$ to $4000 \mathrm{gm}$} & \multicolumn{3}{|c|}{ more than $4001 \mathrm{gm}$} \\
\hline & \multicolumn{3}{|c|}{50} & & 130 & \multicolumn{3}{|c|}{30} \\
\hline
\end{tabular}

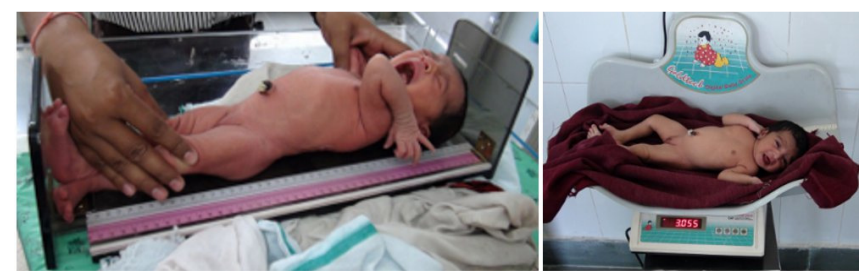

Figure 3 (a).Infantometer Figure 3(b).Weighting machine

\section{FUSION OF EAR AND SOFT BIOMETRIC INFORMATION}

In the proposed framework, the biometric recognition system is divided into two subsystems. The two subsystems are the primary biometric system which consist of ear and the secondary biometric system consisting of soft biometric traits like height, weight, gender and blood-group. Figure 4 shows the architecture of a personal recognition system that makes use of both ear and soft biometric measurements [28,29]. Similar to [17] fusion of ear and soft biometric is performed. 
Signal \& Image Processing : An International Journal (SIPIJ) Vol.3, No.3, June 2012

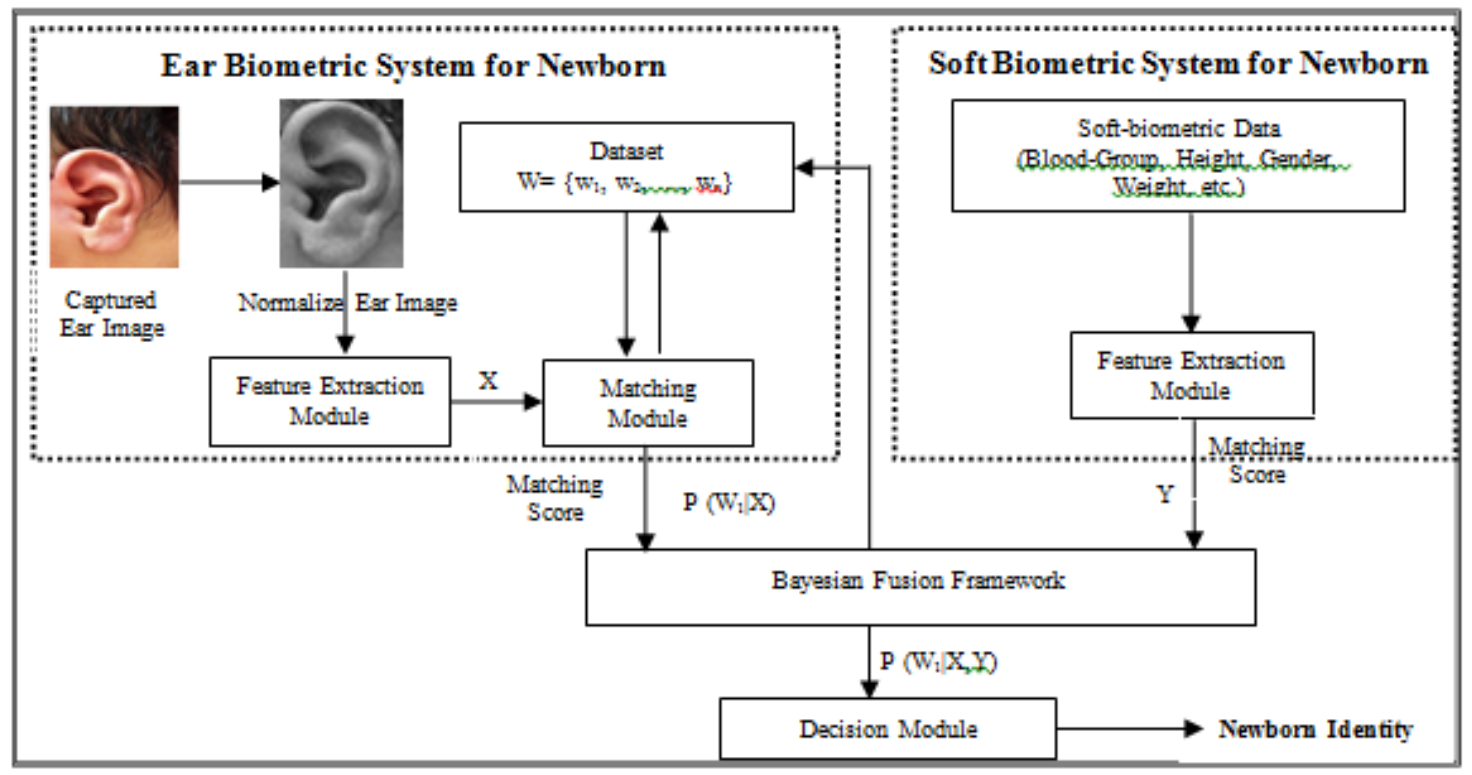

Figure 4. Framework for fusion of primary and soft biometric information. Here $\mathrm{x}$ is the primary (ear) feature vector and $y$ is the soft biometric feature vector.

Let $x=\left[\omega_{1}, \omega_{2}, \ldots, \omega_{n}\right]$ where the total no of infants enrolled is $n$ and $x$ is the feature vector corresponding to the face. The primary biometric system output is the form of $P\left(\omega_{i} \mid x\right), i=1,2, \ldots, n$, where $P\left(\omega_{i} \mid x\right)$ is the probability, $x$ is the feature vector for the test user $\omega_{i}$. The primary biometric system output is a matching score, which is converted into posteriori probability. For the secondary biometric system, we can consider $P\left(\omega_{i} \mid x\right)$ as the prior probability for the corresponding test user $\omega_{i}$.

Let $y=\left[y_{1}, y_{2}, \ldots \ldots y_{k}, y_{k+1}, y_{k+2}, \ldots ., y_{m}\right]$ be the feature vector of soft biometric, where, $y_{1}$ through $y_{k}$ denotes continuous variables and $y_{k+1}$ through $y_{m} \square$ are discrete variables. Finally the matching probability of the user $\omega_{i}$, and the given primary biometric and soft biometrics feature vector is $x$ and $y$, i.e., $P\left(\omega_{i} \mid x, y\right)$ can be calculated using the Bayes' rule as $[28,29,17]$ :

$$
P\left(\omega_{i} \mid x, y\right)=\frac{p\left(y \mid \omega_{i}\right) P\left(\omega_{i} \mid x\right)}{\sum_{i=1}^{n} p\left(y \mid \omega_{i}\right) P\left(\omega_{i} \mid x\right)}
$$

If the variables are independent then equation (1) can be rewritten as follows

$$
P\left(\omega_{i} \mid x, y\right)=\frac{p\left(y_{1} \mid \omega_{i}\right) \ldots p\left(y_{k} \mid \omega_{i}\right) P\left(y_{k+1} \mid \omega_{i}\right) \ldots \ldots p\left(y_{m} \mid \omega_{i}\right) P\left(\omega_{i} \mid x\right)}{\sum_{i=1}^{n} p\left(y_{1} \mid \omega_{i}\right) \ldots p\left(y_{k} \mid \omega_{i}\right) P\left(y_{k+1} \mid \omega_{i}\right) \ldots \ldots p\left(y_{m} \mid \omega_{i}\right) P\left(\omega_{i} \mid x\right)}
$$

In equation (2), $p\left(y_{j} \mid \omega_{i}\right), j=1,2, \ldots, k$ represents the conditional probability of the continuous variable $y_{j}$ for the correspondinguser $\omega_{i}$. This can be evaluated from the conditional density of the variable $j$ for the user $\omega_{i}$. On the other hand, discrete probabilities $p\left(y_{j} \mid \omega_{i}\right), j=k+1, k+$ $2, \ldots$, mrepresents the probability that user $\omega_{i}$ is assigned to the class $y_{j}$. This is a measure of the accuracy of 
the classification module in assigning user $\omega_{i}$ to one of the distinct classes based on biometric indicator $y_{j}$.

The logarithm of $P\left(\omega_{i} \mid x, y\right)$ in equation (2) can be expressed as

$$
\begin{aligned}
\log P\left(\omega_{i} \mid x, y\right)= & \log p\left(y_{1} \mid \omega_{i}\right)+\cdots+\log p\left(y_{k} \mid \omega_{i}\right)+\log P\left(y_{k+1} \mid \omega_{i}\right)+\ldots . . \\
& +\log P\left(y_{m} \mid \omega_{i}\right)+\log P\left(\omega_{i} \mid x\right)-\log p(y)
\end{aligned}
$$

where $p(y)=\sum_{i=1}^{n} p\left(y_{1} \mid \omega_{i}\right) \ldots p\left(y_{k} \mid \omega_{i}\right) P\left(y_{k+1} \mid \omega_{i}\right) p\left(y_{m} \mid \omega_{i}\right) P\left(\omega_{i} \mid x\right)$

The resultant weight in the following discriminant function for infant $\omega_{i}$ as [28]:

$$
\begin{aligned}
& \mathrm{g}_{\mathrm{i}}(\mathrm{x}, \mathrm{y})=\mathrm{a}_{0} \log P\left(\omega_{i} \mid \mathrm{x}\right)+\mathrm{a}_{1} \log p\left(y_{1} \mid \omega_{i}\right)+\mathrm{a}_{\mathrm{k}} \log p\left(y_{k} \mid \omega_{i}\right)+\mathrm{a}_{\mathrm{k}+1} \log P\left(y_{k+1} \mid \omega_{i}\right) \\
& \quad+\mathrm{a}_{\mathrm{m}} \log p\left(y_{m} \mid \omega_{i}\right)
\end{aligned}
$$

where $\sum_{i=0}^{m} \mathrm{a}_{\mathrm{i}}=1$ and $\mathrm{a}_{0} \gg \mathrm{a}_{\mathrm{i}}, \mathrm{i}=1,2, \ldots \mathrm{m}$.

Note: - For the soft biometric traits and primary biometric identifier assigned weights are the $a_{i}$ 's, $\mathrm{i}=1,2, \ldots \mathrm{m}$ and $a_{0}$ respectively.

In order to achieve our goal to extract features from ear we evaluate well-known, classical algorithms: PCA, KPCA, FLDA, ICA,GF and HAAR.

- Principal Component Analysis (PCA) [30,31,32]

- Kernel Principal Component Analysis (KPCA) [33]

- Fisher Linear Discriminant Analysis (FLDA) [34,35,36]

- Independent Component Analysis (ICA) [37,38]

- Geometrical Feature Extraction (GF) [39, 40]

- $\operatorname{HAAR}[41,42]$

Evaluation process is performed five times for checking validation and computed rank-1 identification accuracies. The overall performance evaluations of all the six algorithms (PCA, KPCA, FLDA, ICA, GF and HAAR) are computed on the newborn ear database. The results of this experiment are compiled in following Table 2 and Figure5, it is observed that the identification accuracy of GF is $83.67 \%$ and HAAR is $85.13 \%$ at Rank-I.

Table 2. Identification Accuracy of the Newborn Database

\begin{tabular}{|l|l|l|l|l|l|l|}
\hline Procedure & PCA & KPCA & ICA & FLDA & GF & HAAR \\
\hline $\begin{array}{l}\text { Identification Accuracy } \\
\text { (Rank-1) }\end{array}$ & $78.56 \%$ & $80.03 \%$ & $71.75 \%$ & $80.57 \%$ & $83.67 \%$ & $85.13 \%$ \\
\hline
\end{tabular}


Signal \& Image Processing : An International Journal (SIPIJ) Vol.3, No.3, June 2012

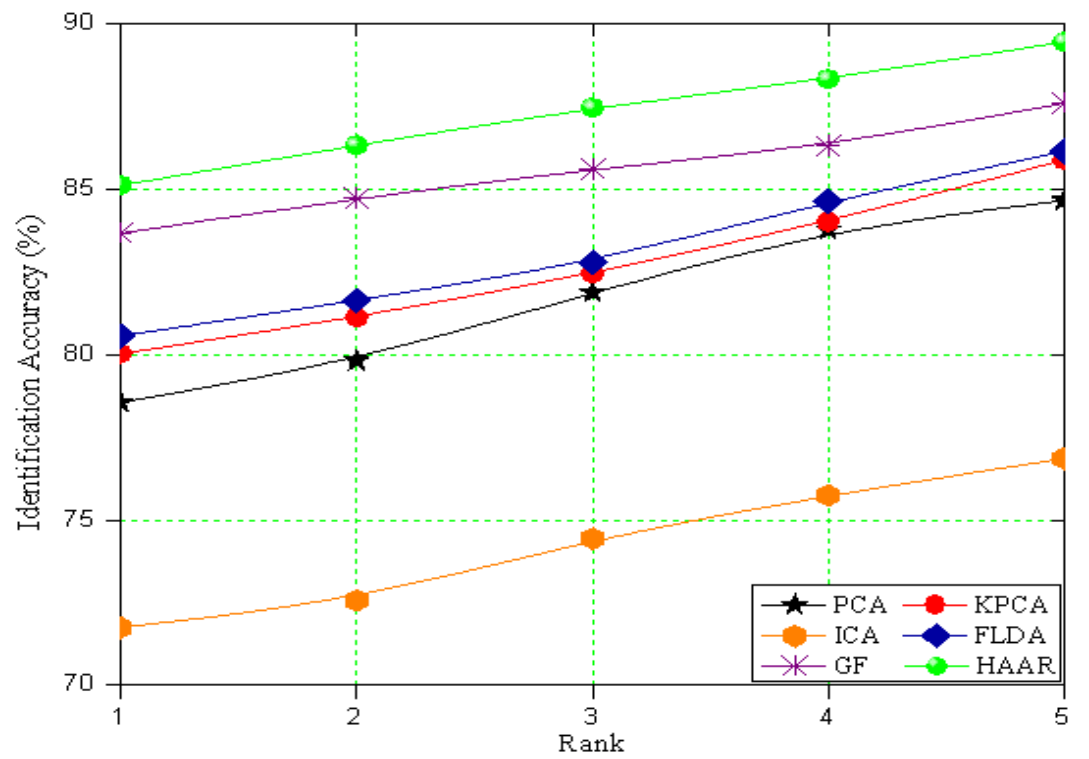

Figure5. CMC for ear recognition algorithm.

In our experiments we have selected soft biometric traits such as gender, blood group,weight, and height information of the user in addition to the ear biometric identifiers.

Let $P\left(\omega_{i} \mid s\right)$ be the posterior probability (Ear) that the user is newborn $\omega_{i} \square$ given the primary biometric score ' $s$ ' of the test user. Let $y_{i}=\left(G_{i}, B_{i}, W_{i}, H_{i}\right)$ is the soft biometric feature vector corresponding to the identity claimed by the user $\omega_{i}$, where $G_{i}, B_{i}, W_{i}$ and $H_{i}$ are the true values of gender, blood group, weight, and height of $\omega_{i}$. Let $y^{*}=\left(G^{*} ; B^{*} ; W^{*} ; H^{*}\right)$ is the soft biometric feature vector of the observed test user, where $G^{*}$ is the observed gender, $B^{*}$ is the observed blood group, $W^{*}$ is the observed weight, and $H^{*}$ is the observed height. Finallythe score after considering the observed soft biometric characteristics is computed as:

$\mathrm{g}_{\mathrm{i}}\left(\mathbf{s}, y^{*}\right)=\mathrm{a}_{0} \log P($ genuine $\mid s)+\mathrm{a}_{1} \log p\left(H^{*} \mid H_{i}\right)+\mathrm{a}_{2} \log P\left(W^{*} \mid W_{i}\right)+\mathrm{a}_{3} \log P\left(G^{*} \mid G_{i}\right) \ldots$

where $\mathrm{a}_{3}=0$,. if $G^{*}=$ "reject", and $\mathrm{a}_{4}=0$ if $B^{*}=$ "reject". $+\mathrm{a}_{4} \log P\left(B^{*} \mid B_{i}\right)$

Figure 5 shows the Cumulative Match Characteristic (CMC) of the ear biometric system operating in the identification mode, and the improvement in performance achieved after the utilization of soft biometric information. The weights assigned to the ear (primary) and soft biometric traits were selected experimentally such that the performance gain is maximized. However, no formal procedure was used and an exhaustive search of all possible sets of weights was not attempted. The use of blood-group, height, weight and gender information along with the ear leads to an improvement of 5.6\% in the rank one performance as shown in Figure6(a), Figure 6(b), Figure 6(c) and Figure6(d)respectively. FromFigure 6(b), Figure 6(c) and Figure6(d), we can observe that the blood-group information of the newborn is more discriminative than gender and leads to a $1.49 \%$ improvement in the rank one performance. The combined use of all the four soft biometric traits results in an improvement of approximately $5.6 \%$ over the primary biometric system as shown in Figure6(e). 
The key analyses of the ear recognition are explained below:-

- The difficulty of ear feature extraction lies in the changes among the same ear caused by head rotation and lighting variation because most of the time newborn are sleeping or crying. The geometry feature extraction depends heavily on the quality of the image preprocessing.

- Due to different lighting conditions the curve segments extraction and the structural extraction will be different even for the same newly born child, which makes the methods unreliable. The rotation discrimination is even more challenging because the angle between the ear and the head is not the same among different babies.

- Table 2 shows that among the appearance based algorithms, FLDA provides the best accuracy of $80.57 \%$ at the Rank-1Level. The performance of appearance based PCA, KPCA, FLDA and ICA algorithm increase with decreasing the size of database decreasing the resolution of the image.

- For Geometrical Feature Extraction (GF) method works on the concept of finding out the points on the contour and distance between them, so the result is approximated in our algorithm by allowing an error of $2 \%$ and accuracy is $83.67 \%$.

- Through experiment, we found that recognition performance of appearance methods (such as PCA, KPCA, FLDA and ICA) will increase dramatically when the input image contains much less background information around the ear.

- HAAR wavelet transform is used to decompose the detected image and compute coefficient matrices of the wavelet which are clustered in the feature template. Decision is made by matching test image with trained image using Hamming distance approach and the result in this algorithm is $85.13 \%$.

Table 3. Identification Accuracy of the Newborn

\begin{tabular}{|l|l|l|l|l|l|c|}
\hline Procedure & E & E + G & E + H & E +W & E + B & E+G+H+W+B \\
\hline $\begin{array}{l}\text { Identification } \\
\text { Accuracy (Rank-1) }\end{array}$ & $85.13 \%$ & $85.18 \%$ & $86.53 \%$ & $86.21 \%$ & $85.26 \%$ & $90.72 \%$ \\
\hline
\end{tabular}

where $\mathrm{E}=$ Ear, $\mathrm{G}=$ Gender, $\mathrm{H}=$ Height, $\mathrm{W}=\mathrm{Weight}, \mathrm{B}=\mathrm{Blood}$-group 
Signal \& Image Processing : An International Journal (SIPIJ) Vol.3, No.3, June 2012

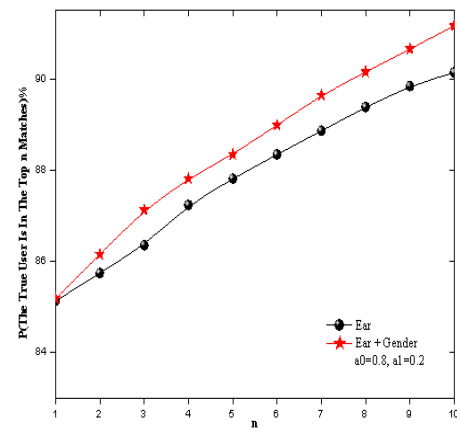

(a)

(b)

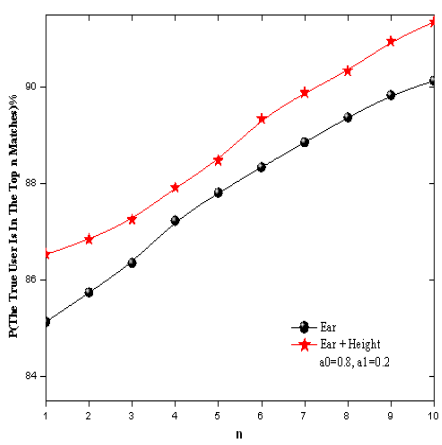

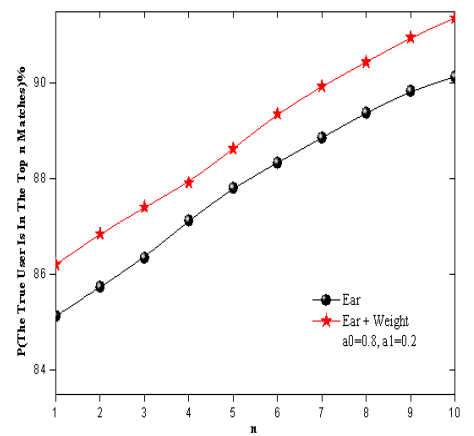

(c)
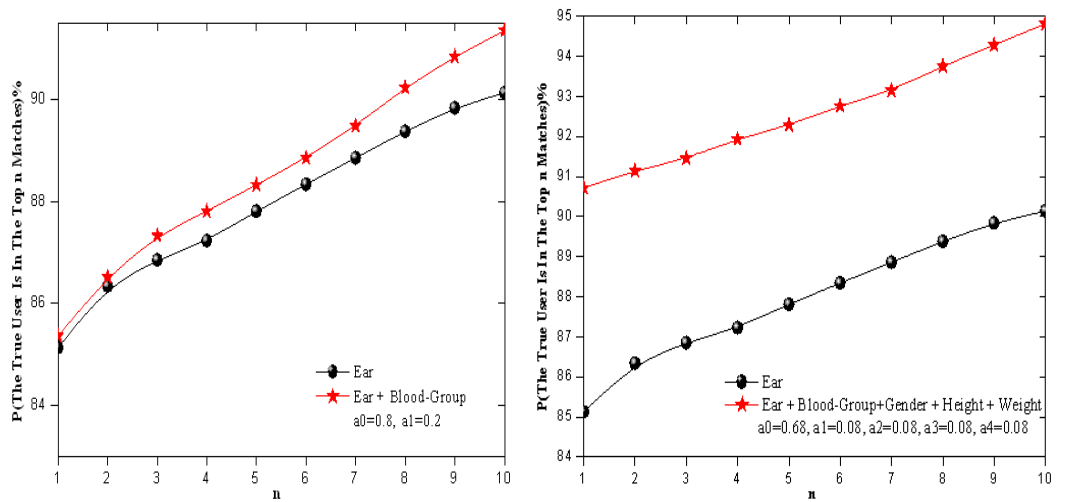

(e)

(d)

Figure 6. Improvement in the performance of a unimodal (Ear) system after addition of soft biometric traits, (a) Ear with Gender, b) Ear with Height, c) Ear with Weight, d) Ear with BloodGroup and e) Ear with Blood-Group ,Gender, Height and Weight

\section{FUTURE DIRECTION}

The approach described in this paper is relatively successful and promising in ear recognition of newborn with fusion of soft biometric data, but more research is to be done by the scientist and engineers in the following domain.

- Size of database is to be increased and following conditions may be considered while capturing images of each subject: illumination, variation, pose, variation, distance variation, date-variation and occlusion variation.

- Collections of ear image of newborn after certain interval of time and then analyze the efficacy of ear recognition in newborn.

- Design and development of pose invariant algorithms as pose is an important covariate in newborn because newborn are highly uncooperative user of biometrics.

- Illumination is also a big challenge because of changing weather condition and the location (indoor or outdoor). So an illumination invariant technique is to be developed.

- In case of newborn occlusion is also a problem as in some case it is found that soon after the birth the ear is pierced and black thread is inserted and some ears are found to be infected by some disease. 
- Ear and Face multimodal biometrics can be used to enhance the identification accuracy and security level.

- To make the enrollment process automatic there is a need to construct a model of variation.

\section{CONCLUSION}

Mixing and kidnapping of newbornis a strong negative response, many parents fear that there is nothing they can do to prevent this tragedy. There is a lot of justification for recognition of newborns using biometrics to mitigate the problem of mixing, switching, abduction and some of the biometric traits collected in the prepared database are justified for only some limited time duration.

The objective of this paper is to demonstrate that ear and soft biometric identifiers such as height, weight, gender, and blood-group can be very useful in newborn recognition. In this research we are getting the accuracy of $85.13 \%$ by using HAAR algorithm and after fusion with soft biometrics the accuracy is $90.72 \%$. It is our assertion that ear and soft biometric data can be a very promising tool for identification of newborn. Although the soft biometric characteristics are not as permanent and reliable as the traditional biometric identifiers like ear, they provide some information about the identity of the newborn that leads to higher accuracy in establishing the user identity.

Our proposed model demonstrated that the utilization of ancillary user information like gender, height, weight and blood-group can improve the performance of the traditional biometric system. Although the soft biometric characteristic are not as permanent and reliable as the traditional biometric identifiers like ear, they provide some information about the identity of the user that leads to higher accuracy in establishing the user identity.

\section{ACKNOWLEDGEMENTS}

Authors would like to thank Prof. B. M. Singh and Dr. Niraj Srivastava (Department of Kaumar Bharitya, Faculty of Aurveda, Institute of Medical Science, BHU, Varanasi-India) for their help and cooperation in preparing the database.

\section{REFERENCES}

[1] http://www.amfor.net/stolenbabies.html, Last accessed on May 25, (2011).

[2] http://www.missingkids.com/enus/documents/infantabductionstats.pdf, Last accessed on June 4, (2011).

[3] J.E. Gray, G. Suresh, R. Ursprung, W.H. Edwards, J. Nickerson, and P.H. Shinno.: Patient Misidentification in the neonatal intensive care unit: Quantification of ris, Paediatrics, vol.117, (2006) e46-e47.

[4] M.E. Stapleton.: Best foot forward: Infant footprints for personal identification, Law Enforcement Bulletin 63, FBI, (1999). 
Signal \& Image Processing : An International Journal (SIPIJ) Vol.3, No.3, June 2012

[5] K.S. Shepard, T. Erickson, and H. Fromm.: Limitations of footprinting as a means of infant identification, Pediatrics, vol. 37, no. 1. (1996).

[6] Thompson, J. E., Clark, D. A., Salisbury, B., and Cahill, J..: Footprinting the infant: not cost-effective, Journal of Pediatrics, (1981) 797-798.

[7] Pela, N. T. R., Mamede, M. V., and Tavares, M. S. G..: Analise critica de impressoes plantares de recem-nascidos, Revista Brasileira de Enfermagem, (1975) 100-105.

[8] Galton, F..: Finger prints of young children,British Association for the Advancement of Science, (1989).

[9] D. Weingaertner, O.R.P. Bellon, M.N.L. Cat, and L. Silva.: Infant's biometric identification: Can it be done?, in International Joint Conference on Computer Vision, Imaging and Computer Graphics Theory and Applications, (2008).

[10]C. Fields, C.F. Hugh, C.P. Warren, and M. Zimberoff.: The ear of the infant as an identification constant, Journal of Obstetrics and Gynecology, vol. 16, (1960) 98-101.

[11] S.Z. Li and A.K. Jain.: Handbook of Face Recognition, Springer, New York, (2004).

[12] J. Daugman.: New methods in iris recognition, IEEE Transactions on Systems, Man and Cybernetics B, vol. 37, no. 5, (2007) 1167-1175.

[13] S. Bharadwaj, H.S. Bhatt, R. Singh, M. Vatsa, and S.K. Singh.: Face Recognition for Infants:A Preliminary Study,Biometrics, Theory Applications and Systems (BTAS), Fourth IEEE International Conference on , vol., no., pp.1-6, (2010) 27-29.

[14] Rubisley P Lemes, Olga R P Bellon, Luciano Silva, Anil K Jain.: Biometric Recognition of Newborns: Identification using Palmprints, International Joint Conference on Biometrics, October 1113, Washington DC USA, (2011).

[15] Shrikant Tiwari, Aruni Singh and Sanjay K Singh.: Newborn's ear recognition: Can it be done?, International Conference on Image Information Processing (ICIIP), 3-5 Nov. JUIT India, 2011.

[16] Shrikant Tiwari, Aruni Singh and Sanjay K Singh.:Can Face and Soft-biometric Traits Assist in Recognition of Newborn?, International Conference on Recent Advanced in Information Technology (RAIT), India, 2012.

[17] Shrikant Tiwari, Aruni Singh and Sanjay K Singh.:Can Ear and Soft-biometric Traits Assist in Recognition of Newborn?, First International Conference on Signal, Image Processing and Pattern Recognition (SPPR 2012), India, 2012.

[18]D. Kuefner, V.M. Cassia, M. Picozzi, and E. Bricolo.: Do all kids look alike? Evidence for anotherage effect in adults, Journal of Experimental Psychology: Human Perception and Performance, vol. 34, no. 4, (2008) 811-817.

[19] Lomuto, C. and Duverges, C..: Identificacion delrecien nacidoy medidas de prevencion para evitar surobo delas maternidades, Revista del Hospital Materno Infantil Ramon Sarda, 14(3):, (1995) 115124.

[20] Alfred Iannarelli.: Ear Identification, Paramont Publishing Company, (1989). 
Signal \& Image Processing : An International Journal (SIPIJ) Vol.3, No.3, June 2012

[21] K.H. Pun and Y.S. Moon.: Recent advances in ear biometrics. In Proceedings of the Sixth International Conference on Automatic Face and Gesture Recognition, pages, (2004) 164-169.

[22] Takaya Yuizono, Yu Wang, Kiminori Satoh, and Shigeru Nakayama.: Study on individual recognition for ear images by using genetic local search, In Proceedings of the 2002 Congress on Evolutionary Computation, pages, (2002) 237-242.

[23] M. Burge and W. Burger.: Ear biometrics in Computer Vision, International Conference of Pattern Recognition, (2000) 822-826.

[24] A. Ross, K. Nandakumar, and A.K. Jain.: Handbook of Multibiometrics, Springer, New York, (2006).

[25] Jain, A.K., Dass, S.C., Nandakumar, K. .:Can soft biometric traits assist user recognition? In: Proceedings of SPIE International Symposium on Defense and Security: Biometric Technology for Human Identification (2004).

[26] Gutta, S., Huang, J.R.J., Jonathon, P.,Wechsler, H.: Mixture of Experts for Classification of Gender, Ethnic Origin, and Pose of Human Faces. IEEE Transactions on Neural Networks 11, 948-960, 2000.

[27] A K Jain, S C Dass, and K Nandakumar.: Soft biometric traits for personal recognition system. In Biometric Authentication. First International Conference, ICBA, (2004) 731-738.

[28] A. K. Jain, K. Nandakumar, X. Lu, and U. Park.: Integrating Faces, Fingerprints and Soft Biometric Traits for User Recognition" In Proceedings of Biometric Authentication Workshop, LNCS 3087, pages 259-269, Prague, Czech Republic (2004).

[29] Jain, A.K., Lu, X..: Ethnicity Identification from Face Image. In: Proceedings of SPIE International Symposium on Defense and Security: Biometric Technology for Human Identification (2004).

[30]P. Belhumeur, J. Hespanha, and D. Kriegman.: Eigenfaces vs. fisherfaces: Recognition using class specific linear projection, IEEE Transactions on Pattern Analysis and Machine Intelligence, vol. 19, no. 7, (1997) 711-720.

[31] Young-Jun Song, Young-Gil Kim, Nam Kim, Jae-Hyeong Ahn.: Face Recognition using both Geometric Features and PCA/LDA, Sixth International Conference on Advanced Language Processing and Web Information Technology.

[32] Y.Ping and K.W.Bowyer.: Empirical Evaluation of Advanced Ear Biometrics, Proc. Empirical Evaluation Methods in Computer Vision, San Diego, (2005) 56-59.

[33] Y.Li,.:Study on Some Key Issues in Ear Recognition, PhD thesis, University of Science and Technology Beijing, Beijing (2006).

[34] Takio Kurita and Toshiharu Taguchi.:A Modification of Kernel-based Fisher Discriminant Analysis for Face Detection.

[35] Wei Liu, Yunhong Wang, Stan Z. Li, Tieniu Tan.: Null Space Approach of Fisher Discriminant Analysis for Face Recognition, Proceeding of ECCV workshop on Biometric Authentication, (2004) $32-44$. 
[36] Li Fen Chen, H.Y. Mark Liao, M.T. Ko, G.J.Yu.: A New LDA-based Face Recognition System Which Can Solve the Small Size Problem, Pattern Recognition, Vol.33, No.10, (2000) 1713 1726.

[37]M.S. Bartlett, J.R. Movellan, and T.J. Sejnowski.: Face recognition by independent component analysis, IEEE Transactions on Neural Networks, vol. 13, no. 6, (2002) 1450-1464.

[38] L. Nanni and A. Lumini.: A multi-matcher for ear authentication, Pattern Recognition. Letter. vol. 28, no. 16 , (2007) 2219-2226.

[39] M. Choras.: Ear biometrics based on geometrical features extraction, Electron. Lett. Comput. Vis. Image Anal., vol. 5, no. 3, (2005) 84-95.

[40] M. Chora's, R.S. Chora's.: Geometrical Algorithms of Ear Contour Shape Representation and Feature Extraction, Proc. of Intelligent Systems Design and Applications (ISDA), IEEE CS Press, vol. II, 451456, Jinan, China, (2006).

[41] Stefan Pittner and Sagar V. Kamarthi.:Feature Extraction from Wavelet Coefficients for Pattern Recognition Tasks, IEEE Transactions on Pattern Analysis and Machine Intelligence, Vol. 21, No. 1, (1999).

[42] Burrus, C., Gopinath, R., and Guo, H.:Introduction to Wavelets and Wavelet Transforms, Prentice Hall, New Jersy, (1998).

\section{Author's Profile:-}

Shrikant Tiwari received his M.Tech. degree in Computer Science and Technology from University of Mysore, India, in 2009. He is currently working toward the PhD degree at the Institute of Technology, Banaras Hindu University, Varanasi, India. His research interests include Biometrics, Image Processing and Pattern Recognition.

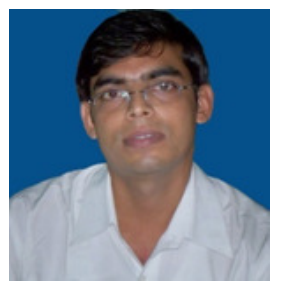

Aruni Singh Assistant Professor in the Department of Computer Engineering, KNIT, Sultanpur, India. His research interests include biometrics, machine learning and pattern classification. Currently he is pursuing Ph.D. at the Institute of Technology, Banaras Hindu University, Varanasi, India.

Sanjay K. Singh is Associate Professor in Department of Computer Engineering at Institute of Technology, Banaras Hindu University, India. He is a certified Novel Engineer and Novel administrator. His research has been funded by UGC and AICTE. He has over 40 publications in refereed journals, book chapters, and conferences. His research interests include computational intelligence, biometrics, video authentication, Pattern recognition and machine learning. Dr. Singh is a member of IET, IEEE, ISTE, CSI.
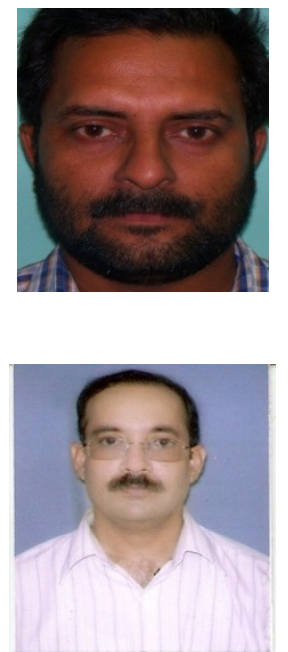